\title{
Assistência ventilatória domiciliar em crianças
}

\author{
Home ventilation of pediatric patients
}

\author{
Eduardo Juan Troster*
}

\begin{abstract}
Oartigo intitulado "Assistência ventilatória domiciliar em crianças - descrição de um programa" de Resener et al., é de grande importância prática. No Brasil, ainda não há uma abordagem padronizada dos pacientes pediátricos que necessitam de suporte ventilatório prolongado. As várias unidades de terapia intensiva pediátrica e/ou neonatal têm uma percentagem variável de pacientes com insuficiência respiratória ventilatória (patologias neuromusculares, problemas ventilatórios de causa central e doenças pulmonares obstrutivas) que necessitam de suporte
\end{abstract} prolongado, tanto no setor público quanto no privado.

Ao redor de $50 \%$ dos internados em unidades de terapia intensiva (UTI) necessitam de ventilação mecânica. Dependendo das afecções respiratórias, os pacientes podem necessitar de suporte ventilatório prolongado $(\geq 21 \text { dias })^{1}$.

Existem várias formas de oferecer ventilação mecânica fora da UTI:

- semi-intensiva (step-down unit);

- centro de desmame regional;

- ventilação mecânica domiciliar.

Há necessidade de que os pacientes estejam estáveis, i.e., sem uso de drogas vasoativas ou parâmetros ventilatórios elevados.

Há também uma pressão das fontes pagadoras para não manter os pacientes em ventilação mecânica prolongada na UTI pelos custos elevados ${ }^{2}$. Na década de 90 houve um incremento na tecnologia disponível para assistência ventilatória domiciliar, propiciando uma segurança maior ${ }^{3}$.

Uma das maiores experiências em ventilação mecânica domiciliar é na França. Uma rede nacional sem fins lucrativos (ANTADIR) tem uma casuística maior do que 26.000 pacientes, tanto em ventilação não invasiva quanto através de traqueostomia ${ }^{4}$.

Com relação à abordagem ventilatória tivemos não invasiva com máscara facial, pressão intermitente (IPPV) através de traqueostomia e uma criança com pressão positiva contínua na via aérea (CPAP). A ventilação mecânica convencional através da traqueostomia está associada com

\footnotetext{
* Doutor em Medicinapelo Depto. de Pediatria-FM USP. Chefe das CTIS Pediátricas do Instituto da Criança Pedro de Alcântara e Hospital Israelita Albert Einstein.
}

complicações infecciosas. A ventilação não invasiva pode ser administrada com pressão positiva ou negativa ${ }^{5}$.

A ventilação não invasiva com pressão positiva foi utilizada com sucesso em crianças com insuficiência respiratória ${ }^{6-8}$. Padman et al. ${ }^{6}$ publicaram uma série de 15 casos com idade de 4 a 21 anos com insuficiência respiratória devido a fibrose cística $(n=4)$ e doença neuromuscular ( $n$ = 11). Em 14 pacientes, foi evitada a instalação de uma via artificial. Houve redução no tempo médio de permanência hospitalar e melhora na freqüência respiratória e cardíaca, nível de bicarbonato, $\mathrm{PaCO}_{2}$, dispnéia e qualidade do sono. Em 1995, Fortenberry et al. ${ }^{7}$ publicaram um estudo retrospectivo sobre a eficácia e as complicações do Bi-PAP em 28 crianças com insuficiência respiratória hipoxêmica aguda. O Bi-PAP reduziu a frequiência respiratória e melhorou a oxigenação e ventilação. O uso do Bi-PAP diminui o tempo médio de permanência hospitalar e melhora o conforto do paciente.

Na discussão do trabalho de Resener et al. neste número do Jornal de Pediatria, nota-se que há um aumento da percentagem dos casos que utilizaram ventilação não invasiva na sua casuística, comparada com a de um trabalho inglês. A facilidade, o conforto, o menor risco de infecções são fatores determinantes que devem estimular seu uso. Entretanto, a seleção dos pacientes é crucial, visto que nem todos os pacientes podem beneficiar-se de uma ventilação com pressão positiva não invasiva ${ }^{5}$.

O Bi-PAP - pressão positiva com 2 níveis (bilevel positive pressure) - é utilizado com IPAP (pressão positiva nas vias aéreas na inspiração) maior do que EPAP (pressão positiva nas vias aéreas na expiração). O Bi-PAP é uma forma de ventilação que é desencadeada pelo paciente, pressão controlada (ventilação com pressão positiva) e ciclado a fluxo ou tempo. Uma opção ventilatória muito indicada para assistência ventilatória domicilar ${ }^{9}$.

Pela necessidade de estabelecer critérios de indicação, recursos necessários (equipamentos e humanos), treinamento adequado (familiar e profissionais na área de Saúde) e a importância da humanização no atendimento, o trabalho de Resener et al. deve servir como modelo para o estabelecimento de um padrão brasileiro. A falta de recursos adequados na área de Saúde é um fator limitante; no entanto a alocação mais racional de recursos deve melhorar a eficiência da assistência ventilatória. 


\section{Referências bibliográficas}

1. Scheinhorn DJ. Stearn-Hassenpflig, M. Provision of long-term mechanical ventilation. Crit Care Med 1998; 14: 819-32.

2. Cohen IL, Booth FVM. Cost containment and mechanical ventilation in the United States. New Horizons 1994; 2: 283.

3. Goldberg AI. Outcomes of home care for life-supported persons: long term oxygen and prolonged mechanical ventilation [Editorial]. Chest 1996; 109: 595.

4. Chailleux E, Fauroux B, Binet F et al. Predictors of survival in patients receiving domiciliar oxygen therapy of mechanical ventilation: a 10 year analysis of ANTADIR observatory. Chest 1996; 109: 741.

5. Venkataraman ST. Noninvasive mechanical ventilation and respiratory care. New Horizons 1999; 7: 353-8.
6. Padman R, Lawless S, Von Nessen S. Use of BiPAP by nasal mask in the treatment of respiratory insufficiency in pediatric patients: preliminary investigation. Pediatr Pulmonol 1994; 17: 119-23.

7. Fortenberry JD, Del Toro J, Jefferson LS et al. Management of pediatric acute hypoxemic respiratory insufficiency with bilevel positive (BiPAP) nasal mask ventilation. Chest 1995: 108: 1059-64.

8. Padman R, Lawless ST, Kettrick RG. Noninvasive ventilation via bilateral positive airway pressure support in pediatric practice. Crit Care Med 1998; 26: 169-73.

9. Pilbeam SP. Home mechanical ventilation. In: Pilbeam SP. Mechanical ventilation. Physiological and clinical applications. $3^{\text {rd }}$ ed. St Louis: Mosby; 1998, p.385-412.

\title{
Refluxo gastroesofágico e doença respiratória na infância
}

\author{
Gastroesophageal reflux and respiratory diseases in children
}

\author{
José Dirceu Ribeiro*
}

\begin{abstract}
Acredita-se que a primeira observação de que desordens respiratórias pudessem estar sob influência do trato digestivo data de 1776, quando Nicholas Rosen von Rosenstein, no seu livro The Diseases of Children and their Remedies, descreveu o que ele chamou de tosse gástrica da infância. Em seguida, William Heberden, em 1802, sugeriu que, em asmáticos, “a respiração é mais curta e mais difícil após uma refeição", e Sir William Osler, em 1892, em seu livro The Principles and Practice of Medicine, relatou que "paroxismos graves de asma podem ser induzidos quando o estômago é sobrecarregado, ou quando usados determinados tipos de alimentos"1.

Entretanto, foi em 1946 que Mendelson provou, em seu estudo clássico sobre aspiração pulmonar durante o ato anestésico, que sintomas sugestivos de obstrução brônquica podem ser causados por aspiração do conteúdo gástrico. Em 1949, pneumonias recidivantes foram observadas por Belcher como complicação de processos esofagianos obstrutivos, levando o nome de "pneumonite por disfagia".
\end{abstract}

\footnotetext{
* Mestre em Clínica Médica, Doutor em Pediatria e Professor de Pediatria da FCM-Unicamp. Vice-presidente do Departamento de Pneumologia da SBP.
}

Finalmente, em 1962, Kennedy abriu uma nova era sugerindo que o refluxo gastroesofágico (RGE) "silencioso ou oculto" pode ser uma causa importante e pouco reconhecida de enfermidades pulmonares. A partir dessa época numerosos estudos clínicos mostraram associação da doença do RGE (DRGE) e uma variedade de sinais, sintomas e doenças do aparelho respiratório ${ }^{1-3}$.

O RGE pode ocorrer de maneira fisiológica em qualquer indivíduo. O termo doença do refluxo gastroesofágico tem sido utilizado para descrever o amplo espectro de distúrbios causados pelo RGE. A distinção entre RGE fisiológico e DRGE é feita em função da freqüência e intensidade do $\mathrm{RGE}^{4}$. No entanto, apesar da associação entre doenças respiratórias e RGE ser bem reconhecida, o significado exato desta relação e suas implicações clínicas ainda permanecem controversos.

A DRGEé uma doença multifatorial, na qual os eventos iniciais ainda são desconhecidos e uma prevalência aumentada de RGE em pneumopatas não indica por si só uma relação causal.

Se em indivíduos saudáveis temos o RGE fisiológico normal, no outro extremo temos indivíduos com esofagite grave. Entre os dois extremos comprovam-se vários graus (intensidade) e tipos (causas) de RGE (funcional, patológico, secundário). 